\title{
Infrared study of high-pressure molecular phases of carbon dioxide
}

\author{
Valentina M. Giordano ${ }^{1,2}$, Federico A. Gorelli ${ }^{1,3}$, and Roberto Bini ${ }^{1,4}$ \\ ${ }^{1}$ LENS, European Laboratory for Non-linear Spectroscopy and INFM \\ Via Carrara 1, Sesto Fiorentino, Firenze I-50019, Italy \\ E-mail: bini@chim.unifi.it \\ 2 IMPMC/Physique des Milieux Denses, CNRS UMR 7590, Université Pierre et Marie Curie \\ 140 rue Lourmel, Paris 75015, France \\ ${ }^{3}$ CRS-SOFT-INFM-CNR, c/o Università di Roma La Sapienza \\ P.zza A. Moro 2, Roma I-00185, Italy \\ ${ }^{4}$ Dipartimento di Chimica dell' Università di Firenze \\ Via della Lastruccia 3, Sesto Fiorentino, Firenze I-50019, Italy
}

Received April 14, 2006

\begin{abstract}
The infrared absorption spectra of the high-pressure crystalline phases II, III and IV of solid $\mathrm{CO}_{2}$ were studied by using a resistive heated diamond anvil cell up to $30 \mathrm{GPa}$. The employment of crystal slabs having thickness of $\sim 2 \mu \mathrm{m}$ allowed the study of the strongly absorbing fundamental bending and antisymmetric stretching modes without saturation. These are the first data for phases II and IV in the fundamental modes spectral region, furthermore the high samples quality allowed to improve, with respect to previous studies, the characterization of the infrared spectra of phases I and III. The comparison of the spectral structure and of the frequency evolution with pressure of the crystal modes between phase I and the higher pressure phases clearly indicates the close resemblance among all these phases. In particular, the dramatic change of the intermolecular interaction claimed for phases II (dimeric association) and IV (large molecular bending) can be ruled out and, as a consequence, the hypothesis of a transition from the molecular phase I to the silica-like phase $\mathrm{V}$ through intermediate nonmolecular phases discarded.
\end{abstract}

PACS: 78.30.-j, 31.70.Ks, 81.30.-t

Keywords: infrared absorption spectra, high-pressure molecular phases, vibrational properties.

\section{Introduction}

The interest in the high-pressure behavior of carbon dioxide arises from several reasons. It is indeed a common component of both interior and atmosphere of the Earth and some giant planets, and it is also employed as a supercritical fluid in organic syntheses. Recently, it was also found that it transforms to a silica-like extended solid at high pressure and temperature. Since the hardness of a compound is strictly connected to the presence in the network of light elements, $\mathrm{CO}_{2}$ becomes an excellent candidate to produce ultrahard materials. This extended covalent solid (commonly labelled as phase V) can be obtained at relatively moderate pressure and temperature values [1], even though the first synthesis of this material was realized at temperatures exceeding $1000 \mathrm{~K}$ [2-4]. Following its discovery, a renewed interest arose for all the high-pressure high-temperature $\mathrm{CO}_{2}$ polymorphs since these phases could be possibly interpreted as intermediate steps in a continuous transformation from the molecular phase I to the extended nonmolecular phase $\mathrm{V}$ [2]. In the quite narrow pressure range below $20 \mathrm{GPa}$ four solid phases have been identified (Fig. 1) among which only phase I, the so-called dry ice, has been claimed to exhibit the typical characteristics of a molecular crystal. The structure of this phase is cubic $(P a \overline{3}, Z=4)$ and it is 


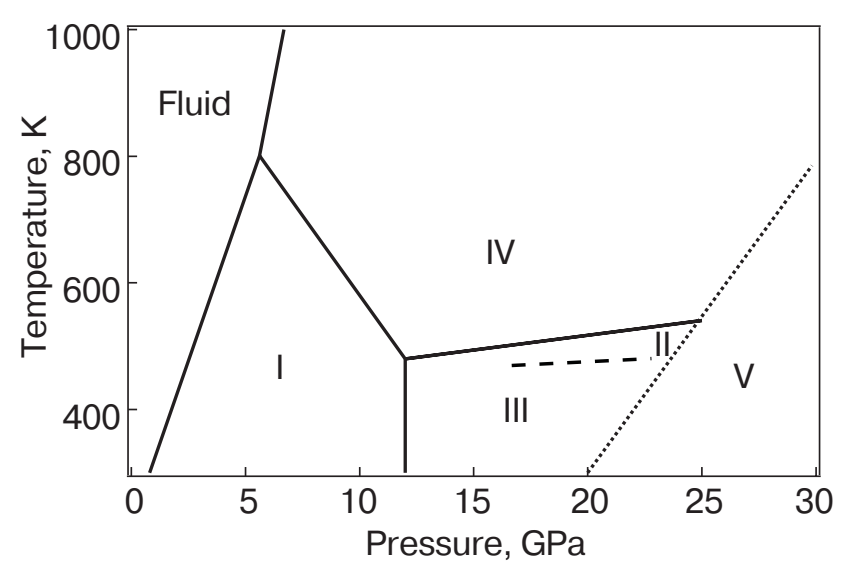

Fig. 1. Phase diagram of $\mathrm{CO}_{2}$ following the studies reported in Refs. 1 and 11. The dashed line is the kinetic barrier between phase III and II, while the dotted line bounds the stability domain proposed by Santoro et al. for phase $\mathrm{V}$.

stable at room temperature between 0.5 and $\approx 11 \mathrm{GPa}$, where it starts transforming with no apparent volume discontinuity to a probably metastable orthorhombic Cmca structure with $Z=2$ (phase III) reported to be stable at least up to $60 \mathrm{GPa}$ [3]. An unusually large bulk modulus (87 $\mathrm{GPa}$ ) has been reported for phase III [3,5]. Above $12 \mathrm{GPa}$ two different phases, II [6] and IV [4], are observed on increasing temperature. The structures of these two phases could not be solved exactly and were suggested to be tetragonal or orthorhombic [7,8]. A strong modification in the intermolecular interactions was proposed for both phases leading to a dimeric association of the molecules in phase II [7], and a large molecular bending in phase IV $[4,8,9]$ as suggested by the Raman activation of the bending mode. These changes were interpreted as a prelude to the breaking of the double $\mathrm{C}=\mathrm{O}$ bond and the formation of new covalent bonds between different molecules occurring in phase $\mathrm{V}$. This interpretation has been recently contested in a DFT calculation by Bonev et al. [10], which claimed a molecular character for all these phases (III, II and IV). Their simulated structures exhibit similar characteristics as phase I, that is, low bulk modulus, intramolecular distances similar to the ambient pressure values, and weak intermolecular interactions. These theoretical results have been substantially confirmed by our recent work on phases II and IV, where the measured infrared and Raman spectra suggest an orthorhombic structure $(Z=4)$ for both phases, provide a better characterization of the threshold temperature of the two transitions, clearly indicate the absence of strong intermolecular interactions and the almost linearity of the molecule in phase IV, and do not show any evidence of a dimeric association in phase II [11]. According to these results the extended covalent solid would apparently be produced without any intermediate stable phase where important molecular distortions or oligomers replacing the monomeric unit act as potential seeds of phase $\mathrm{V}$.

In this work we present a detailed infrared absorption study of phases I, II, III, and IV. By using thin samples (thickness $\sim 2 \mu \mathrm{m}$ ) we could measure the molecular fundamental vibrations without saturation. Our data are the first ones reported for phases II and IV and by their high spectral quality they represent an improvement in the knowledge of the infrared spectrum of phase III. The comparison between the spectral features of all the studied phases allows to investigate the changes in the intermolecular interaction and definitely rule out the hypothesis of the existence of intermediate steps prior to the formation of phase V.

\section{Experimental details}

Liquid $\mathrm{CO}_{2}$ (99.99\% purity) was loaded in a membrane diamond-anvil cell (MDAC) equipped with IIa diamonds at 6 bar and $220 \mathrm{~K}$ by a cryoloading technique. In order to study the fundamental bending $\left(v_{2}\right)$ and asymmetric stretching $\left(v_{3}\right)$ modes avoiding saturation the sample was prepared according to the following procedure. The gasket hole $(150 \mu \mathrm{m}$ diameter $)$ was almost completely filled with a $\mathrm{KBr}$ pellet before the $\mathrm{CO}_{2}$ loading. $\mathrm{KBr}$ was chosen because it does not have any infrared mode in the frequency regions of interest to the present experiment. Comparison among experiments performed with and without $\mathrm{KBr}$ demonstrates that it is also chemically inert with respect to $\mathrm{CO}_{2}$ in the $P-T$ range investigated. The thickness of the $\mathrm{CO}_{2}$ thin layer was estimated by comparing the absorption intensity of the $v_{1}+v_{3} ; 2 v_{2}+v_{3}$ combination bands measured both in the bulk, of known thickness $(\sim 40 \mu \mathrm{m})$, and in the thin samples. A thickness of $\sim 2 \mu \mathrm{m}$ resulted from this procedure. A ruby chip was used to measure the pressure [12]. An external resistive heater able to generate temperatures as high as $650 \mathrm{~K}$ was used to produce the high-temperature phases II and IV and to improve the crystalline quality by several annealings. Infrared absorption spectra were collected by a FT-IR Bruker IFS-120 HR spectrometer modified for performing measurements on samples in the DAC $[13,14]$. MCT or InSb detectors were employed in combination with a $\mathrm{KBr}$ beam splitter. In both cases the experimental resolution was better of $1 \mathrm{~cm}^{-1}$. 


\section{Results}

We performed several experiments to measure the infrared internal vibrations of solid phases I, II, III, and IV. In a typical run, room temperature phase I was compressed in order to obtain phase III, which was successively heated to produce phase II and phase IV. These latter were therefore quenched to room temperature and infrared spectra were collected during decompression. The procedure to obtain phases II and IV has been carefully described in a work [11]. Briefly, to obtain a high quality phase III the sample should be annealed at $20 \mathrm{GPa}$ above $400 \mathrm{~K}$, in fact only at this temperature the characteristic lattice phonon infrared spectrum is observed. By further heating the phase III crystal above $465 \mathrm{~K}$, only the IR active lattice phonons of phase II are observed. Here the transition can be also appreciated by visual observation for the formation of the polycrystalline morphology characteristic of this phase [3]. Phase IV is typically obtained just above $500 \mathrm{~K}$ at $20 \mathrm{GPa}$. Its detection is facilitated by the high transparency of the sample, by the splitting of the $v_{1}+v_{3} ; 2 v_{2}+v_{3}$ combination bands and by the appearance of the very characteristic lattice phonon spectrum composed by five bands.

Representative spectra collected on the four solid phases in the bending region are reported in Fig. 2. The very high quality of the absorption spectrum measured in phase I, nicely resembling room pressure low temperature data obtained on a thin crystalline layer [15], should be remarked. This is a clear confirmation of the reliability of our method to produce very thin crystalline slabs in the DAC. The spectral features measured in phase I have been easily indexed

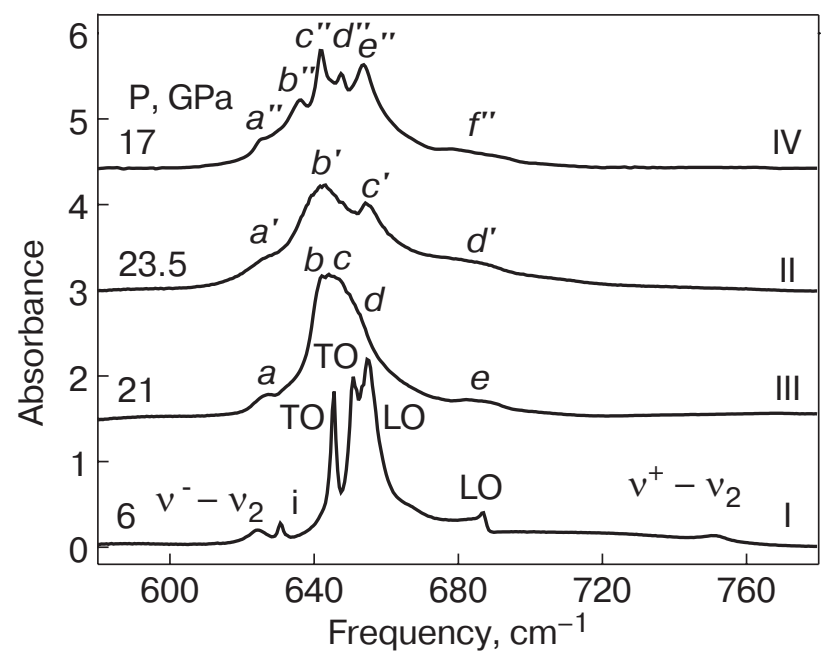

Fig. 2. Representative IR spectra of phases I, II, III, and IV measured at room temperature in the bending region on a thin sample. For the details of the assignment reported for phase I see text. following the room pressure assignment reported by Dows and Schettino [15]. As it can be seen in Fig. 2, we are able to identify all the bending components of phase I reported at room pressure and low temperature with the only exception of the two weaker isotopic peaks due to ${ }^{18} \mathrm{O}^{12} \mathrm{C}^{16} \mathrm{O}$ and ${ }^{18} \mathrm{O}^{13} \mathrm{C}^{16} \mathrm{O}$ [15], thus achieving a relevant advance with respect to previous high pressure studies $[16,17]$. Specifically, we observe the two hot bands $v^{-}-v_{2}$ and $v^{+}-v_{2}(624.3$ and $\left.752 \mathrm{~cm}^{-1}\right)$, the ${ }^{16} \mathrm{O}^{13} \mathrm{C}^{16} \mathrm{O}$ isotopic peak $\left(630.6 \mathrm{~cm}^{-1}\right)$, and the four bending components, expected on the basis of symmetry requirements and LO-TO splitting: 645.6, 650.9, 654.7, and $687 \mathrm{~cm}^{-1}$ at $6 \mathrm{GPa}$. A linear extrapolation of the frequency evolution with pressure (see Fig. 5) fits nicely the ambient pressure values reported by Dows et al. [15]. From this comparison it comes out that the lowest frequency band of the main triplet was erroneously assigned to the ${ }^{18} \mathrm{O}^{12} \mathrm{C}^{16} \mathrm{O}$ isotopic peak while it is evident that represents the lowest TO mode. As a consequence one of the two central peaks that were assigned as the two transverse modes is the second LO component.

The employment of the thin crystal slab is particularly important for the study of the antisymmetric stretching mode $\left(v_{3}\right)$ which has the strongest absorption in the IR spectrum. Characteristic spectra measured in the four different phases are reported in Fig. 3. Phase I spectra, although resembling those reported by Lu et al. [17], allow the LO and TO components to be identified. Other two weak peaks are observed at $\sim 2325 \mathrm{~cm}^{-1}$ (see inset in Fig. 3). The most intense has been identified as the asymmetric stretch-

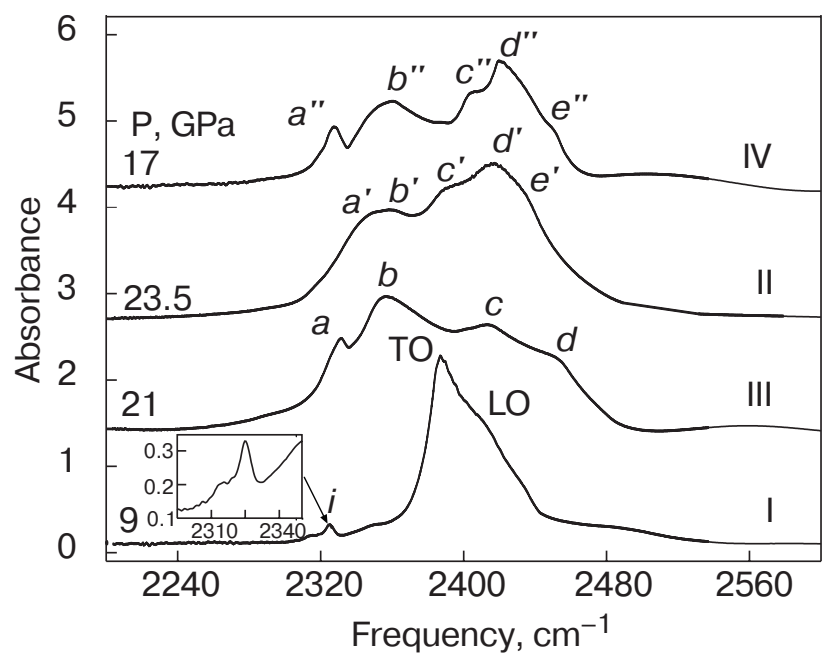

Fig. 3. Representative IR spectra of phases I, II, III, and IV measured at room temperature in the stretching region on a thin sample. The assignment reported for phase I is from Ref. 15. In the inset a zoom of the isotopic stretching peaks in phase I is shown. 
ing mode of the isotope ${ }^{13} \mathrm{C}^{16} \mathrm{O}_{2}$, while the other one was reported as belonging to the isotope ${ }^{16} \mathrm{O}^{13} \mathrm{C}^{18} \mathrm{O}$ [15]. However, the intensity of the latter peak is much higher than expected on the basis of the natural abundances of the ${ }^{18} \mathrm{O}$ isotope. A possible alternative explanation can be provided taking into account the interaction between two ${ }^{13} \mathrm{C}^{16} \mathrm{O}_{2}$ molecules. Because of its abundance, there is a non-zero probability that two nearest neighbor molecules have this isotopic composition. Following the procedure described in Ref. 18, we could fit the intensity profile of the doublet with a distribution law based on this probability and we verified that the minor peak is indeed due to the interaction between two isotopic molecules.

The last spectral region of interest to this study is due to the $v_{1}+v_{3} ; 2 v_{2}+v_{3}$ combination modes at $\sim 3800 \mathrm{~cm}^{-1}$. The IR spectrum of this region has been extensively studied so far only in phase I $[15,16,19]$. In this phase the absorption spectrum consists basically of two narrow absorption peaks (see Fig. 4) at 3633 and $3763.5 \mathrm{~cm}^{-1}$, being the latter rather strong. These two peaks were ascribed to the simultaneous excitation of the two modes $v_{3}$ and $v_{1}$ (or $2 v_{2}$ ) on the same molecule [15], while two-molecule excitation was indicated to be responsible of the high frequency shoulder observed for both peaks. Following the arguments reported in Ref. 20 and concerning the assignment of the biphonon plus phonon modes in the $3 v_{2} ; v_{1}+v_{2}$ region, these peaks may be interpreted as due to the combination of the $v^{+}(\mathbf{k})$ and $v^{-}(\mathbf{k})$ modes $\left(v_{1} ; 2 v_{2}\right.$ Fermi resonance region) with the asymmetric stretching mode $v_{3}(-\mathbf{k})$. The $v^{+}\left(v^{-}\right)$and $v_{3}$ modes are rather different as the frequency dispersion of the wavevector $\mathbf{k}$ is concerned, being the formers extremely localized [21]. By neglecting the dispersion of the $v^{+}$and $v^{-}$modes we summed their frequency

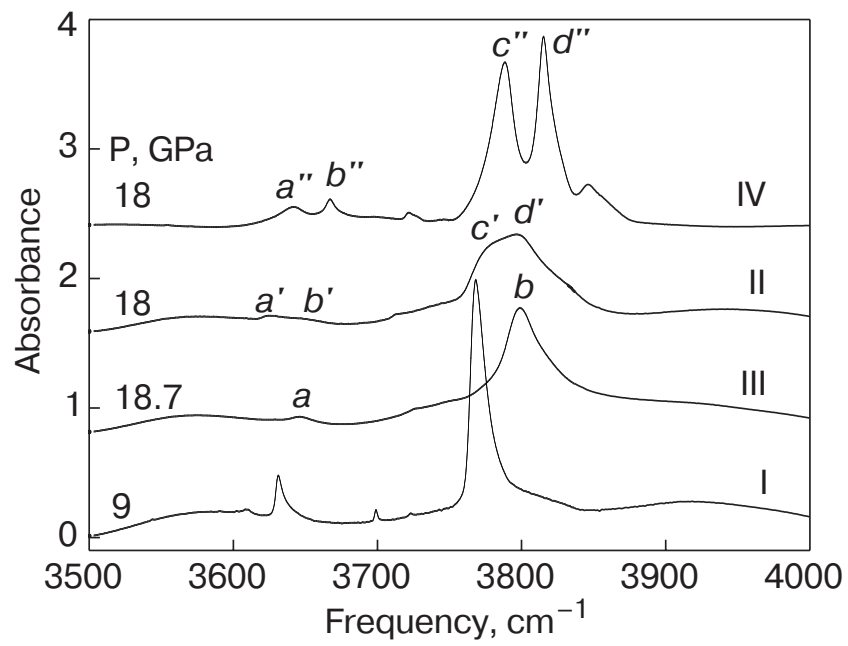

Fig. 4. Representative IR spectra of phases I, II, III, and IV measured at room temperature in the $v_{1}+v_{3}, 2 v_{2}+v_{3}$ combinations region.

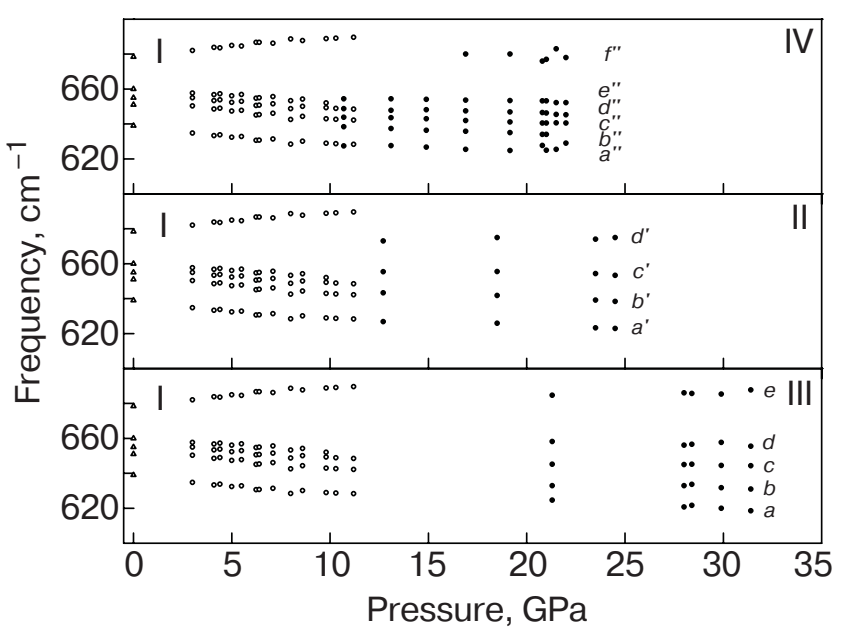

Fig. 5. Pressure shift of the bending components in phase I $(\mathbf{O})$ and in the high pressure phases $(\bullet)$. Room pressure low temperature values $(\triangle)$ from Ref. 15 are also reported for comparison. Starting from the bottom the data for phases III, II, and IV are reported, respectively.

values (by Refs. 16, 22) to the measured IR absorption pattern of $v_{3}$ in the bulk sample under the assumption that the latter is partly resembling the corresponding density of states. With this procedure a good agreement, as the frequency dispersion is concerned, between the observed and calculated absorption pattern is found at any pressure. Therefore we can assign the two main peaks as $v^{-}+v_{3}$ and $v^{+}+v_{3}$ in order of increasing frequency.

In the IR spectra of phase III we could identify 5 and 4 peaks in the bending and stretching regions, respectively (see Figs. 2 and 3), while only two broad and asymmetric peaks, labelled $a$ and $b$ in Fig. 4, are observed in the $v^{-}+v_{3}$ and $v^{+}+v_{3}$ region. The relative intensity of these peaks resembles that of phase I and only the high frequency band $b$ can be detected as the pressure is further increased. The spectral results reported here represent a relevant advance with respect to the previous work of Lu et al. [17]. These authors employed diluted carbon dioxide in argon in order to reduce the absorption of the fundamental modes. However, despite the hydrostatic conditions of the experiment, the spectral quality was poorer than in the present work and only few components were detected. This comparison remarks once more the reliability of the employment of thin crystalline layers also for studies performed above $20 \mathrm{GPa}$.

As the high-pressure phases II and IV are concerned, this is the first complete analysis of the mid infrared region. We have identified up to 5 stretching peaks for both the phases, and 4 and 6 bending absorption bands in phase II and IV, respectively. The spectra in the combination regions are more complex 
than in phase III, with the splitting of both the main peaks. This splitting is common to both phases, and it is better resolved in phase IV. It should be remarked that the sharp doublet $\left(3750-3800 \mathrm{~cm}^{-1}\right)$ measured in phase IV in the $v^{+}+v_{3}$ region represents a characteristic signature of this phase.

Some of the spectral features were easily assigned by comparison with phase I spectra. The lowest frequency peak in the bending and stretching regions (peaks $a, a^{\prime}$ and $a^{\prime \prime}$ ) was assigned for all the phases to the isotopic peak due to ${ }^{13} \mathrm{C}^{16} \mathrm{O}_{2}$. Furthermore, the highest frequency component $\left(e, d^{\prime}\right.$ and $f^{\prime \prime}$ for the bending mode and $d, e^{\prime}$ and $e^{\prime \prime}$ for the antisymmetric stretching mode) may be assigned to the highest frequency LO component, with reference to phase I. The hot bands $v^{-}-v_{2}$ and $v^{+}-v_{2}$ observed in the bending region in phase I are visible in the high-pressure phases only in the thick $(\sim 40 \mu \mathrm{m})$ samples.

Finally, the number of crystal components observed for the bending and antisymmetric stretching modes is reported in Table 1, along with the predictions based on symmetry requirements and LO-TO splitting for all the phases. It should be remarked that the crystal structures indicated for phases II and IV have been selected, among those proposed following $\mathrm{x}$-ray diffraction studies, on the basis of the full vibrational information [11]. All the expected stretching components are observed in phases II and IV, while some $v_{2}$ components are missing likely masked by the line broadening.

Table 1. Comparison between the observed bending and stretching components and those expected for the high-pressure phases on the basis of symmetry requirements and LO-TO splitting.

\begin{tabular}{c|c|c|c|c}
\hline \hline Phase & Space group & Mode & Predicted & Observed \\
\hline \hline \multirow{2}{*}{ III } & Cmca, $Z=2$ & $v_{2}$ & 6 & 4 \\
& & $v_{3}$ & 4 & 3 \\
II & Pnnm, Z=4 & $v_{2}$ & 6 & 3 \\
& & $v_{3}$ & 4 & 4 \\
IV & Pbcn, Z $=4$ & $v_{2}$ & 6 & 5 \\
& $($ Cmca $)$ & $v_{3}$ & 4 & 4 \\
\hline \hline
\end{tabular}

\section{Discussion}

The comparison of the IR absorption spectra of phase I, that is definitely molecular, with the other phases has shown extensive similarities in all the spectral regions investigated. This resemblance contrasts with the thesis of a transformation to the extended silica-like solid driven by the formation of intermediate nonmolecular phases. Both the dimeric association

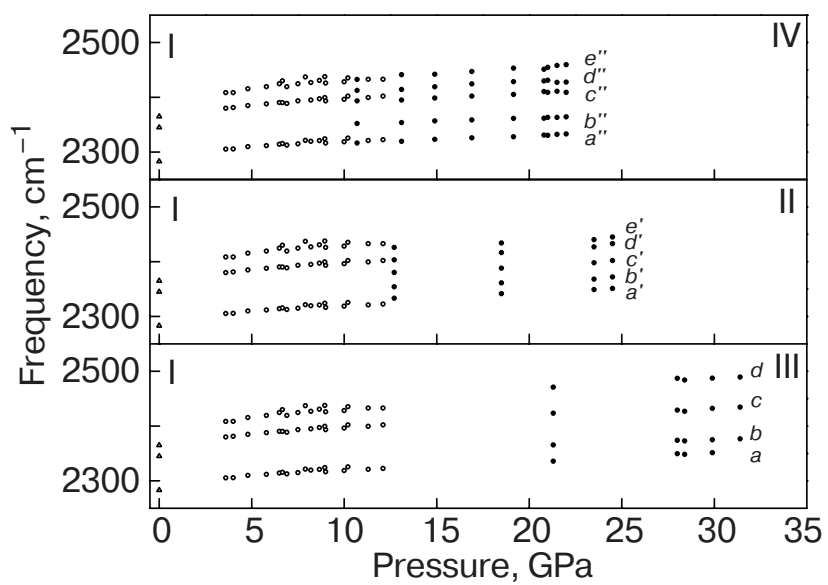

Fig. 6. Pressure shift of the stretching components in phase I (O) and in the high pressure phases $(\mathbf{O})$. Room pressure low temperature values $(\triangle)$ from Ref. 15 are also reported for comparison. Starting from the bottom the data for phases III, II, and IV are reported, respectively.

suggested for phase II and the large molecular bending $\left(C O C \sim \sim 160-170^{\circ}\right)$ claimed for phase IV should definitely determine severe spectral changes. In fact, in phase II an elongation of the $\mathrm{C}-\mathrm{O}$ bond greater than $12 \%$ with respect to phase I, and a drop of the shortest intermolecular contacts to $2.38 \AA$ is claimed [7]. On the other hand, the large molecular bending occurring in phase IV should be accompanied by a bond lengthening to $\sim 1.5 \AA$ and by a huge decrease of the intermolecular distances to $2.1 \AA[8]$. A further indication of the molecular character of all the phases can be gained by the analysis of the pressure shift of all the observed peaks. For each spectral region analyzed in the present work we report how the peak frequen-

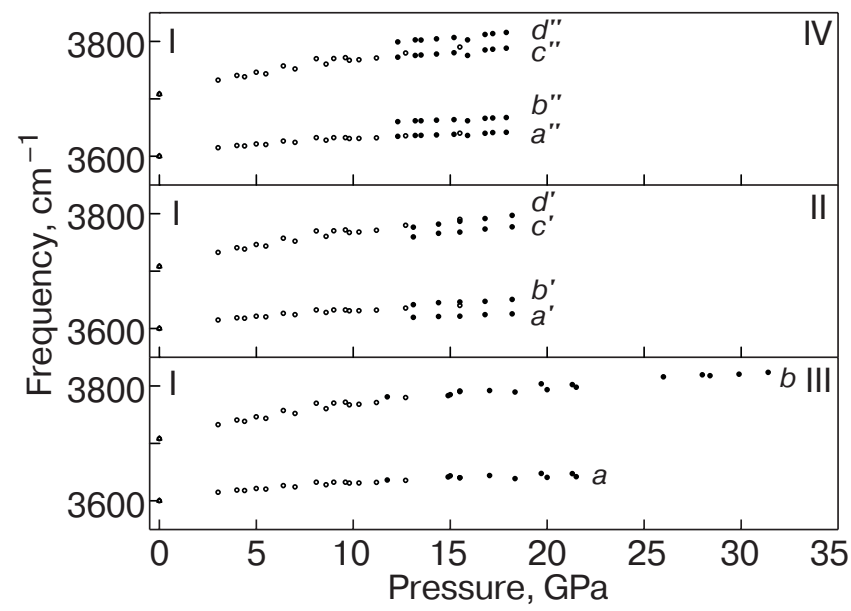

Fig. 7. Pressure shift of the $v^{-}+v_{3}$ and $v^{+}+v_{3}$ peaks in phase I (O) and in the high pressure phases $(\mathbf{O})$. Room pressure low temperature values $(\triangle)$ from Ref. 15 are also reported for comparison. Starting from the bottom the data for phases III, II, and IV are reported, respectively. 
cies behave with pressure in the four different phases (Figs. 5, 6, 7). The frequency data relative to phase I are shown as a kind of reference indicating the molecular pressure shift. With the only exception of phase I, only unloading data are reported because each measurement was performed releasing pressure until the sample transformed to phase I. The comparison between the data relative to phase I and to the other phases shows that a general continuity is always observed in all the studied regions, thus confirming a not significant change of the intermolecular interactions in this pressure range.

More in detail, the softening of the bending mode with pressure was reported as a peculiar characteristic of phase I likely correlated to a structural instability. However, it was reproduced in simulations taking into account a mainly dipolar inter-molecular interaction, with both non-polarizable and polarizable dipoles [23]. The observation of a very similar softening in all the high-pressure phases provides a clear indication of a profound analogy of the involved interactions. It is worth noticing that the red shift of the bending components in phase IV is even less important than in phase I (Fig. 5). Besides suggesting the molecular character of the phase, this is also important in supporting our previous conclusion [11] of a negligible bending of the molecule in this phase. In fact, a bending angle as large as given by Park [8] would cause the transformation of a vibrational degree of freedom to a rotational one, thus implying a bending component to become a lattice mode. In this case we should observe a strong softening of at least one bending component. Similarly, there is no hint of the elongated intra-molecular distances and reduced inter-molecular distances suggested for phase II by Yoo et al. [7], which would cause a significant change in the vibrational frequencies.

Also the analysis of the $v^{-}+v_{3}$ and $v^{+}+v_{3}$ combination region supports a close resemblance of all the phases as the intermolecular interactions are concerned. First, the pressure shift of the main combination bands shows a clear continuity with respect to phase I, thus suggesting a very similar Fermi interaction between $2 v_{2}$ and $v_{1}$ in all the phases. As a matter of fact the bands $a$ and $b$ in phase III correspond to the excitations $v^{-}(\mathbf{k})+v_{3}(-\mathbf{k})$ and $v^{+}(\mathbf{k})+v_{3}(-\mathbf{k})$, respectively, with reference to phase $\mathrm{I}$. The same assignment can be readily extended to phases II and $\mathrm{IV}$, and the splitting into two components is just due to the crystalline field, as expected on the basis of symmetry requirements. Therefore, the bands $a^{\prime}, b^{\prime}$ (phase II) and $a^{\prime \prime}, b^{\prime \prime}$ (phase IV) may be assigned as two components of $v^{-}+v_{3}$, while $c^{\prime}, d^{\prime}$ (phase II) and $c^{\prime \prime}, d^{\prime \prime}$ (phase IV) of $v^{+}+v_{3}$. The same splitting is also expected, but not observed, in phase III.

\section{Conclusions}

We have reported an extended infrared study of the high-pressure molecular phases of carbon dioxide. By producing very thin samples we have been able to measure the fundamental vibrations $v_{2}$ and $v_{3}$ without saturation. The high quality of our spectra allowed to improve the characterization of phase III by resolving more components than previously reported, and to provide the first infrared data on phases II and IV.

Our results indicate a strong similarity among the vibrational properties of all the high-pressure phases and those of phase I. As anticipated in Ref. 11, this means that the molecular geometry as well as the intramolecular and intermolecular distances does not change significantly with respect to phase I, thus ruling out the claimed molecular dimeric association in phase II and the large bending in phase IV, which should cause dramatic spectral changes not observed in this work.

Recently, it was demonstrated in the case of benzene, another very stable molecular system which reacts to give an extended solid, that the molecular instability is driven, within the same crystal phase, by the pressure and temperature tuning of the intermolecular distances [24]. In analogy with these results, the formation of the extended covalent network characteristic of phase $\mathrm{V}$ can be be better viewed as a dynamical process due to a complex interplay between changes in the electronic structure and in the intermolecular distances rather than to the formation of nonmolecular phases acting as a kind of structural intermediates that already contain the seed of the extended covalent silica-like solid.

This work has been supported by the European Union under Contract RII3-CT2003-506350, by the Italian Ministero dell'Università e della Ricerca Scientifica e Tecnologica (MURST) and by «Firenze Hydrolab» through a grant by Ente Cassa di Risparmio di Firenze.

1. M. Santoro, J.F. Lin, H.K. Mao, and R.J. Hemley, J. Chem. Phys. 121, 2780 (2004).

2. V. Iota, C.S. Yoo, and H. Cynn, Science 283, 1510 (1999).

3. C.S. Yoo, H. Cynn, F. Gygi, G. Galli, V. Iota, M. Nicol, S. Carlson, D. Hausermann, and C. Mailhiot, Phys. Rev. Lett. 83, 5527 (1999).

4. V. Iota and C.S. Yoo, Phys. Status Solidi B223, 427 (2001)

5. K. Aoki, H. Yamawaki, M. Sakashita, Y. Gotoh, and K. Takemura, Science 263, 356 (1994). 
6. V. Iota and C.S. Yoo, Phys. Rev. Lett. 86, 5922 (2001).

7. C.S. Yoo, H. Kohlmann, H. Cynn, M.F. Nicol, V. Iota, and T. LeBihan, Phys. Rev. B65, 104103 (2002).

8. J.H. Park, C.S. Yoo, V. Iota, H. Cynn, M.F. Nicol, and T. LeBihan, Phys. Rev. B68, 014107 (2003).

9. C.S. Yoo, V. Iota, and H. Cynn, Phys. Rev. Lett. 86, 444 (2001).

10. S.A. Bonev, F. Gygi, T. Ogitsu, and G. Galli, Phys. Rev. Lett. 91, 065501 (2003).

11. F.A. Gorelli, V.M. Giordano, P.R. Salvi, and R. Bini, Phys. Rev. Lett. 93, 205503 (2004).

12. H.K. Mao, P.M. Bell, J.V. Shaner, and D.J. Steinberg, J. Appl. Phys. 49, 3276 (1978).

13. R. Bini, R. Ballerini, G. Pratesi, and H.J. Jodl, Rev. Sci. Instrum. 68, 3154 (1997).

14. F. Gorelli, L. Ulivi, M. Santoro, and R. Bini, Phys. Rev. Lett. 83, 4093 (1999).

15. D.A. Dows and V. Schettino, J. Chem. Phys. 58, 5009 (1973).
16. R.C. Hanson and L.H. Jones, J. Chem. Phys. 75, 1102 (1981)

17. R. Lu and A.M. Hofmeister, Phys. Rev. B52, 3985 (1995).

18. P. Loubeyre, R. LeToullec, and J.P. Pinceaux, Phys. Rev. B45, 12844 (1992).

19. K. Aoki, H. Yamawaki, and M. Sakashita, Phys. Rev. B48, 9231 (1993)

20. R. Bini, P.R. Salvi, V. Schettino, and H.J. Jodl, Phys. Lett. A157, 273 (1991).

21. F. Bogani and P.R. Salvi, J. Chem. Phys. 81, 4991 (1984)

22. H. Olijnyk, H. Daufer, and H.J. Jodl, J. Chem. Phys. 88, 4204 (1988).

23. G. Cardini, P.R. Salvi, V. Schettino, and H.J. Jodl, J. Chem. Phys. 91, 3869 (1989).

24. L. Ciabini, M. Santoro, F.A. Gorelli, R. Bini, V. Schettino, and S. Raugei, Nature Mater., to be updated. 